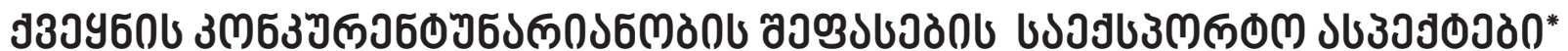

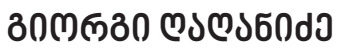

https://doi.org/10.35945/gb.2018.06.003

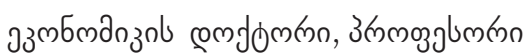

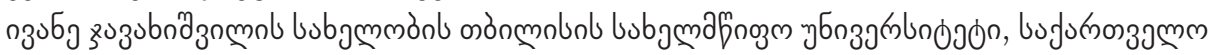

giorgi.gaganidze@tsu.ge

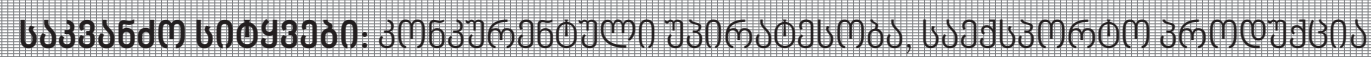

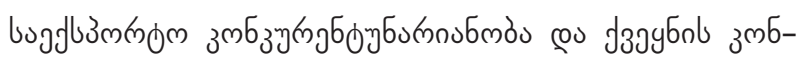

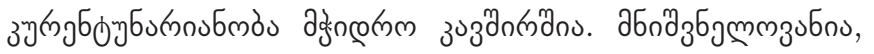

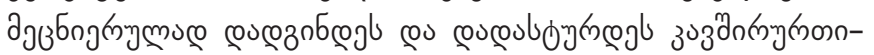

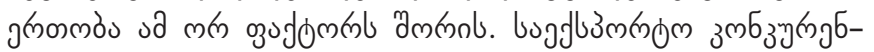

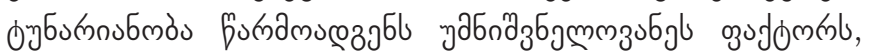

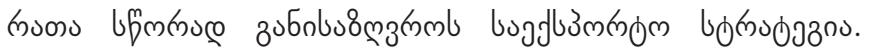

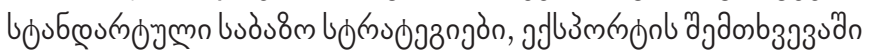

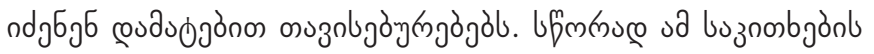
zu

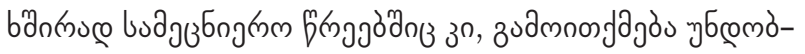

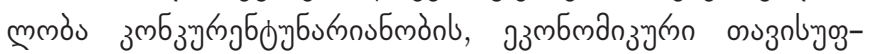

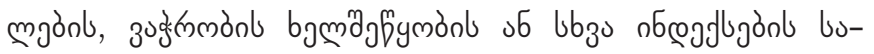

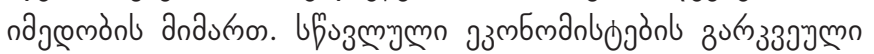

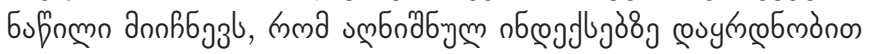

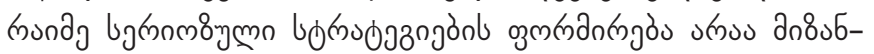

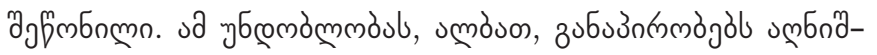

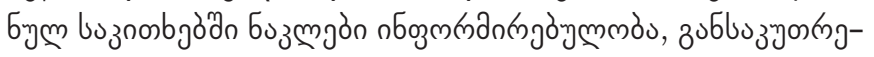

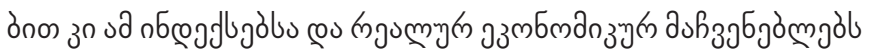

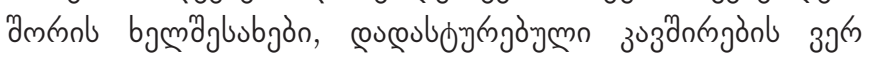

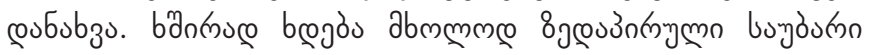

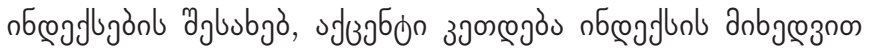

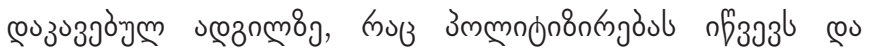

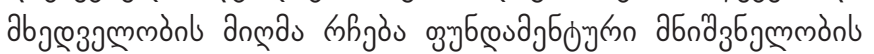

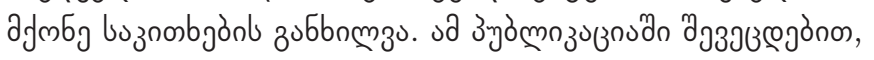

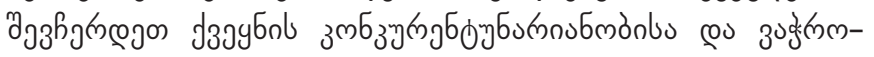

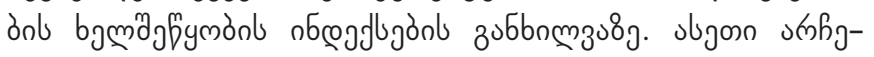

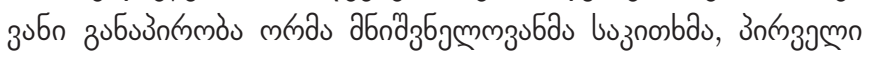

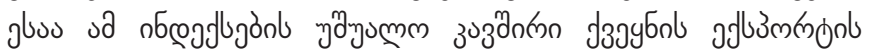

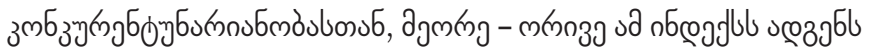

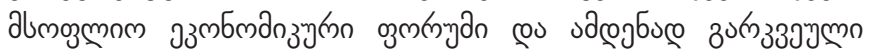

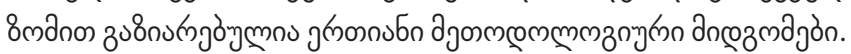

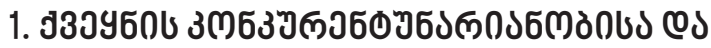

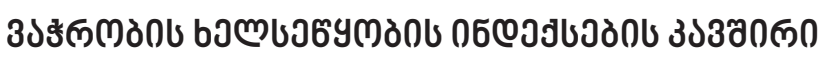

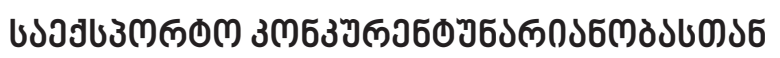

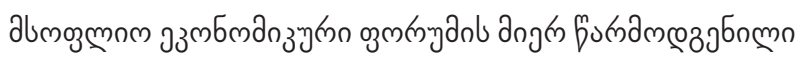

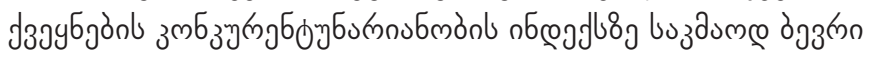

"ззмлз

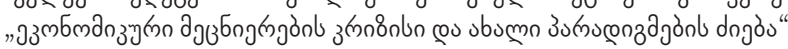

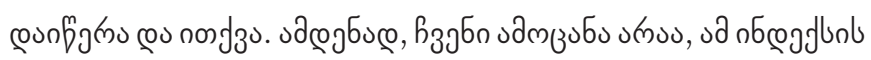

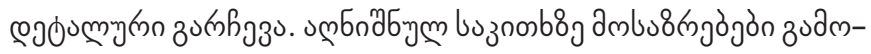

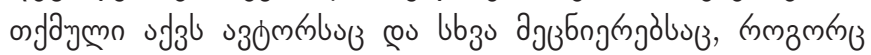

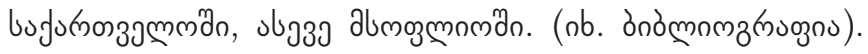

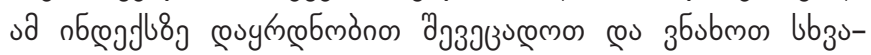

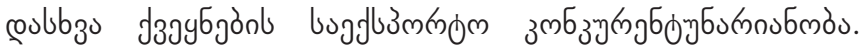

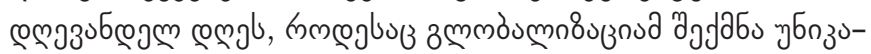

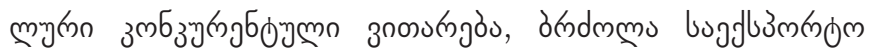

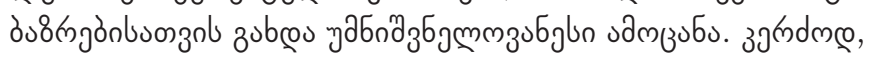

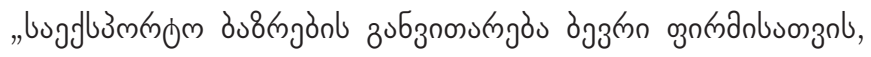

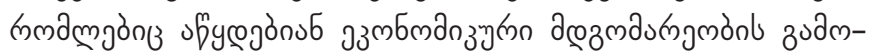

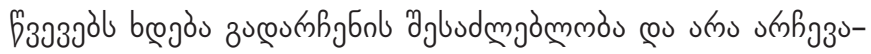

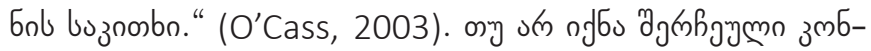

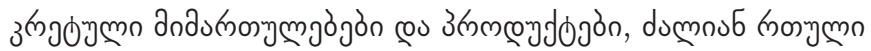

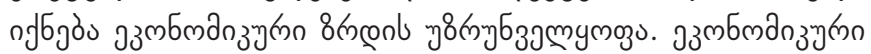

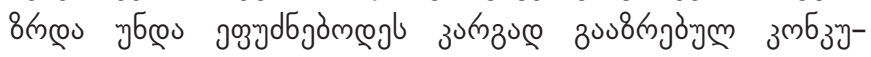

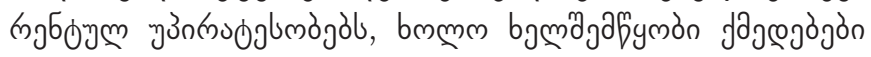

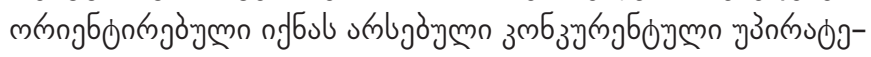

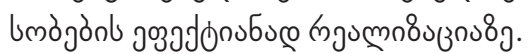

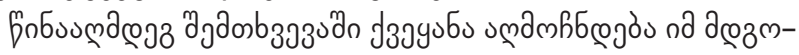

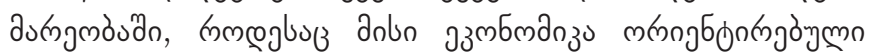

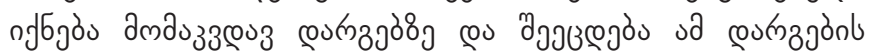

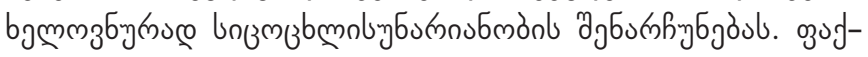

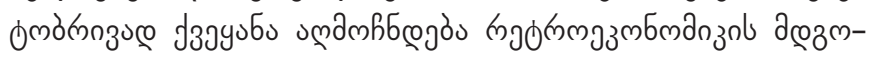

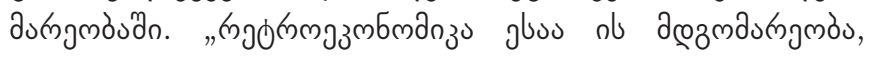

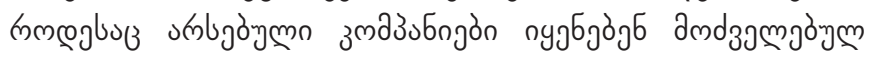

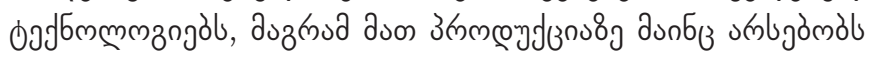

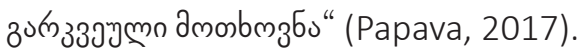

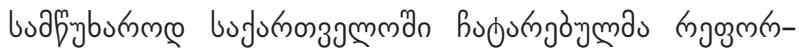
a

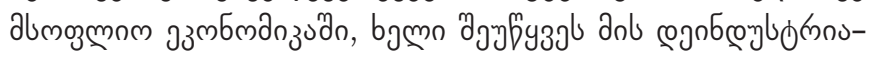

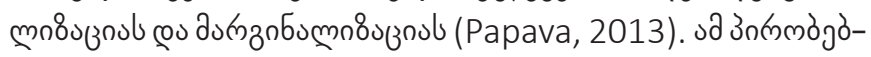

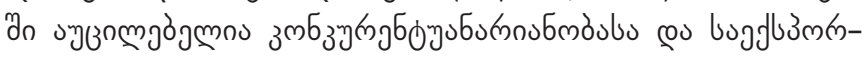

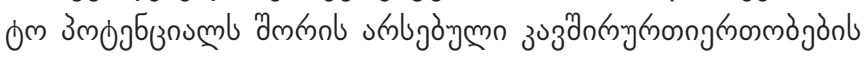

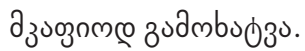

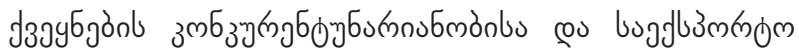

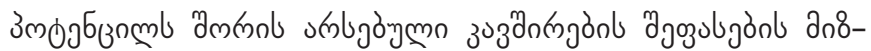

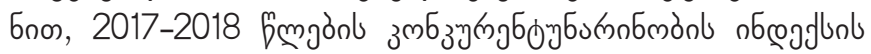

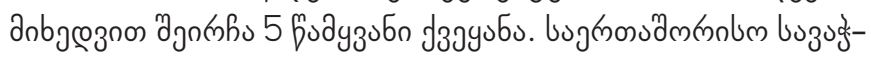

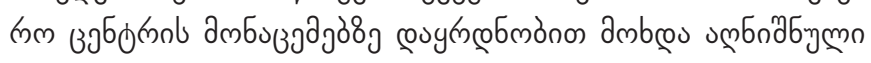




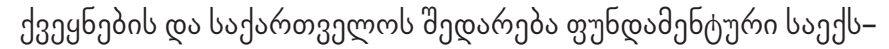

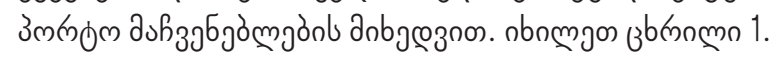

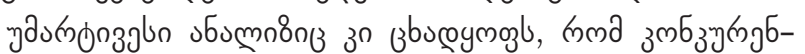
oचб

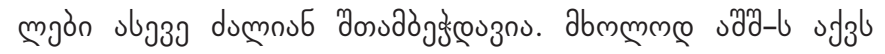

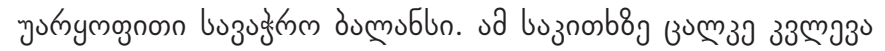

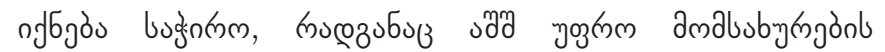

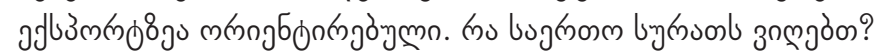

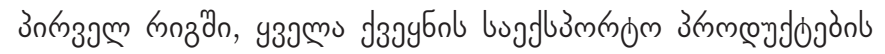

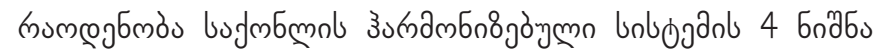

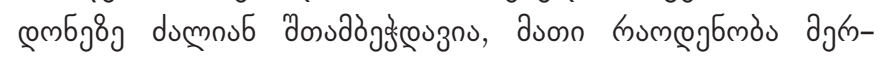

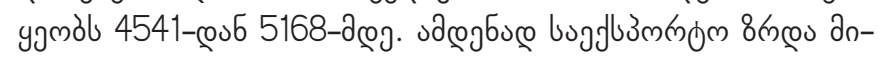

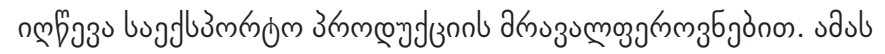

2013a; Gaganidze, 2013b; Gaganidze, 2013c; Silagadze\& Zubiashvili, 2015; Silagadze\&Atanelishvili, 2014).

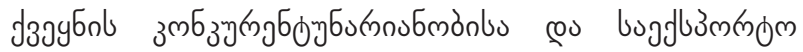
auh 3 бб

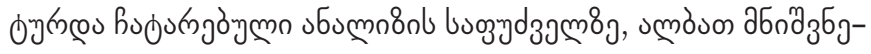

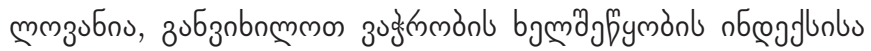

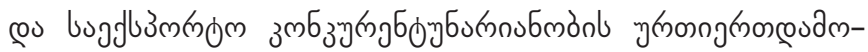

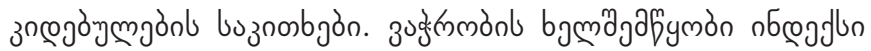

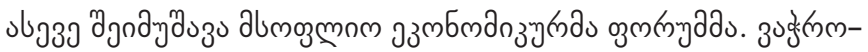

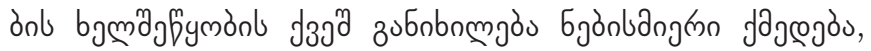

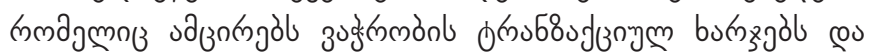

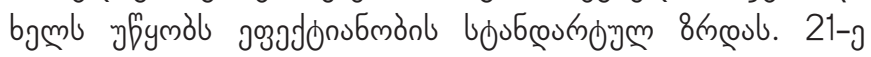

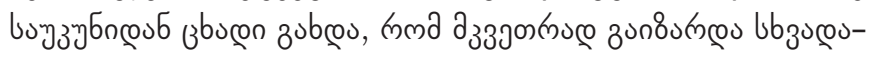

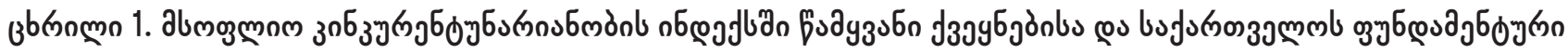

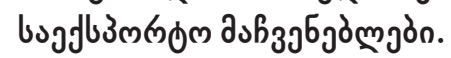

\begin{tabular}{|c|c|c|c|c|c|c|c|}
\hline 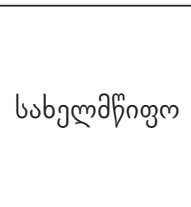 & 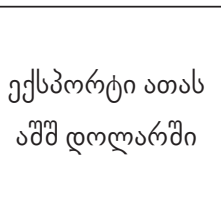 & 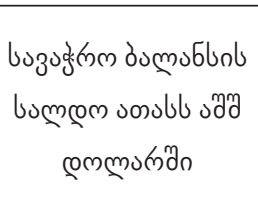 & $\begin{array}{l}\text { aflumminonb } \\
\% \text { alumozmom } \\
\text { aflummong }\end{array}$ & 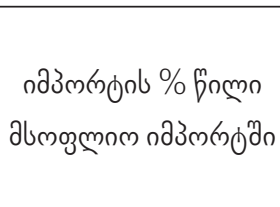 & 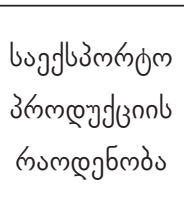 & 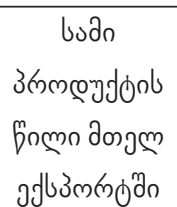 & 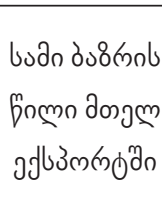 \\
\hline$y_{39 n g 3 \text { mon }}$ & 304688646 & 35533861 & 1,89 & 1.64 & 4541 & 46,2 & 37,2 \\
\hline səz & 1453169633 & -796491086 & 9,00 & 13,73 & 5168 & 15,6 & 42,2 \\
\hline unбzo3ynn & 329868096 & 46862251 & 2,04 & 1,73 & 4620 & 25,2 & 36,2 \\
\hline Ӟmmsб боро & 569376621 & 65196526 & 3,53 & 3,08 & 4985 & 18,0 & 41,5 \\
\hline 8ogk & 1340751954 & 280079536 & 8,3 & 6,47 & 4950 & 13,3 & 24,3 \\
\hline bufornoszgmm & 2113469 & 5121169 & 0,01 & 0,04 & 1735 & 30,9 & 26,0 \\
\hline
\end{tabular}

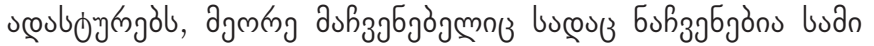

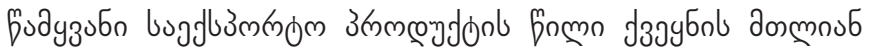

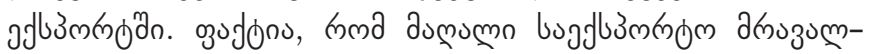

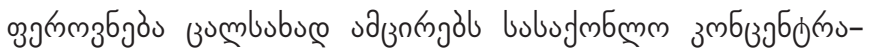

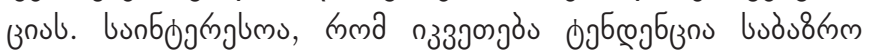

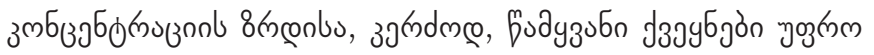

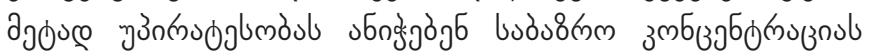

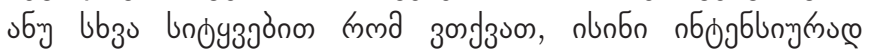

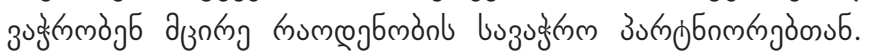

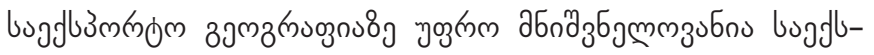

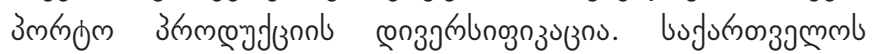

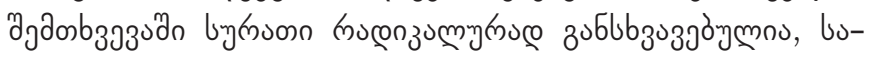

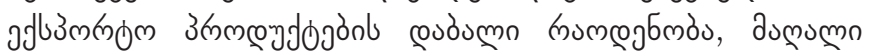
bubuनg

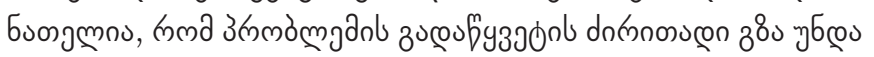

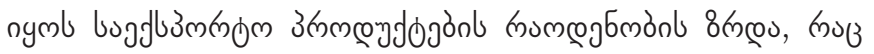

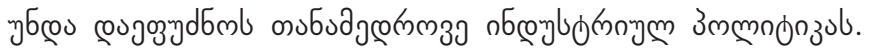

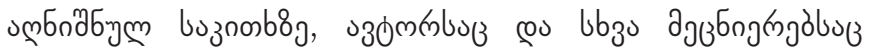

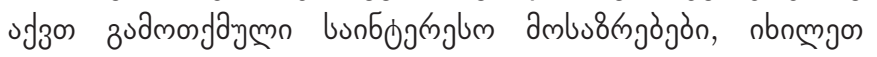

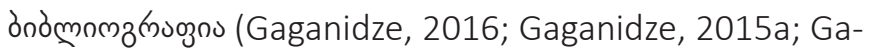
ganidze, 2015b; Gaganidze, 2014a; Gaganidze, 2014b; Gaganidze, 2014c; Gaganidze, 2014d; Gaganidze,

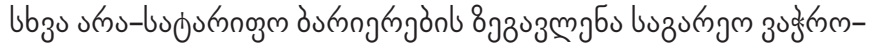

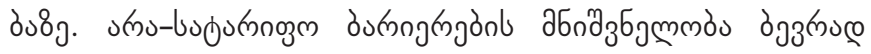

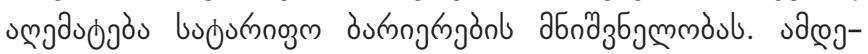

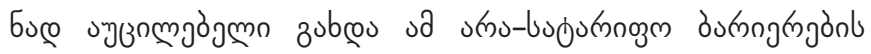

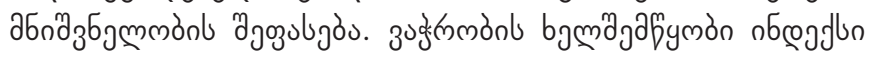

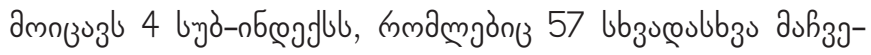

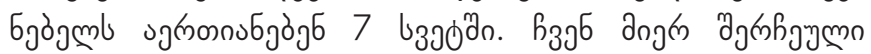

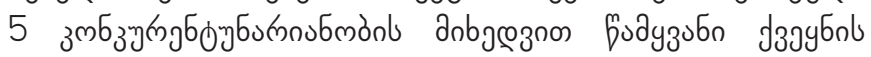

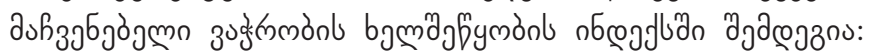

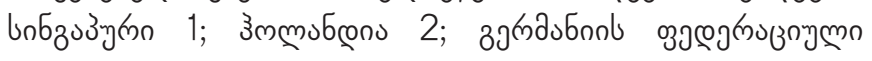

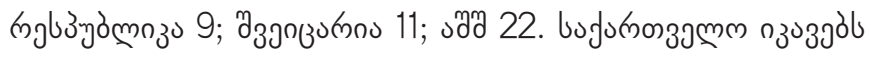

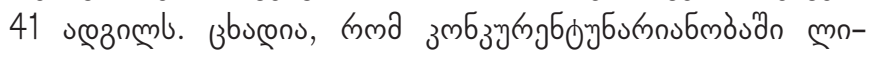

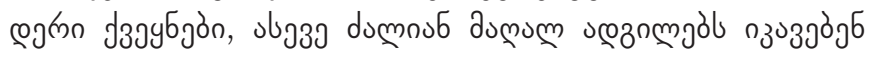

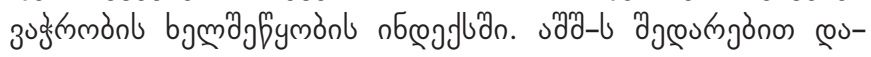

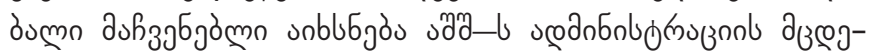

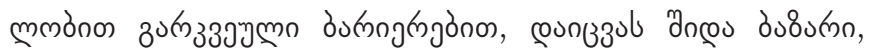

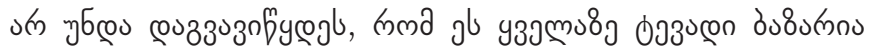

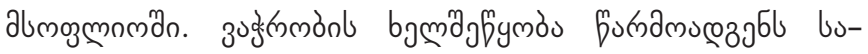

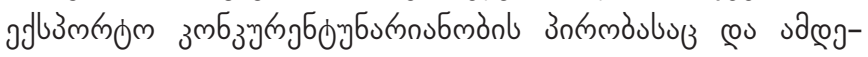

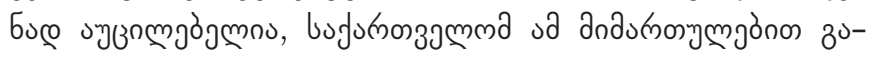

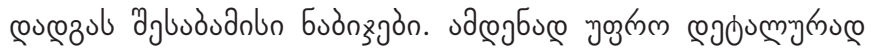

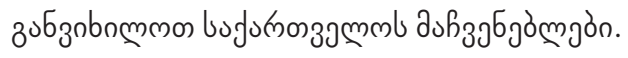




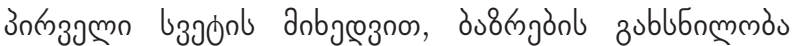

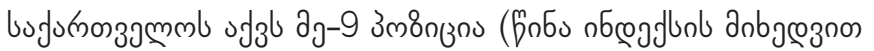

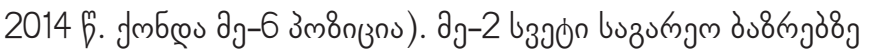

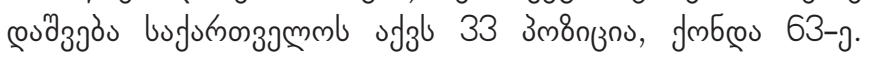

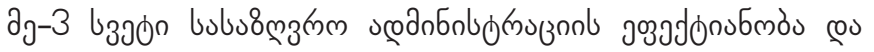

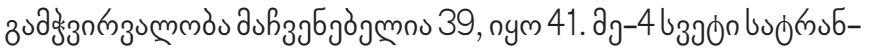

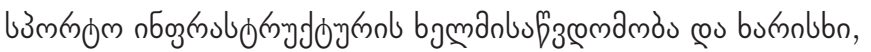

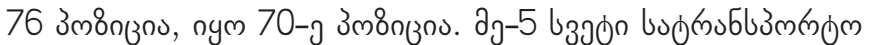

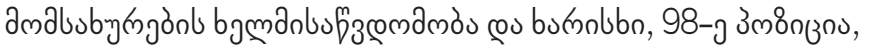

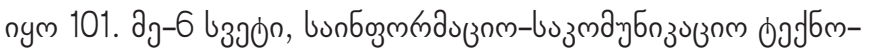

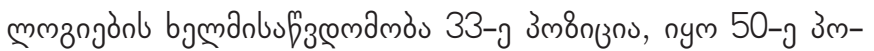

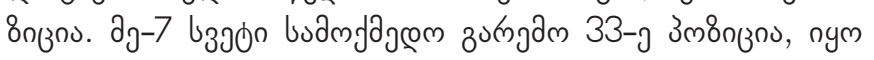

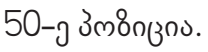

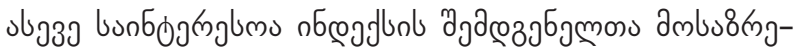

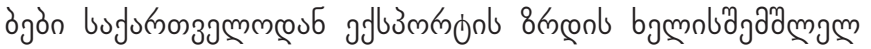

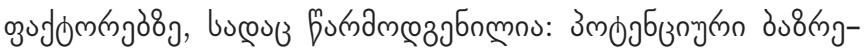

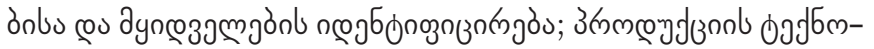

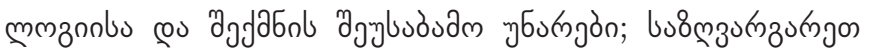

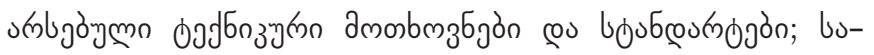

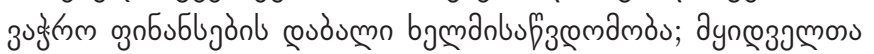

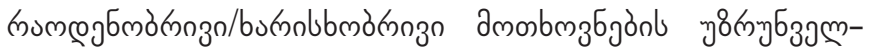

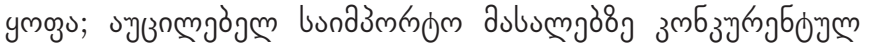

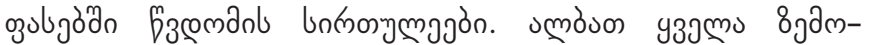

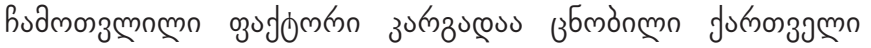

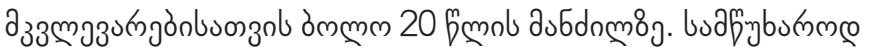

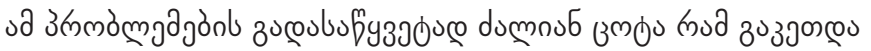

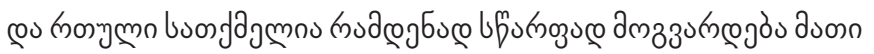

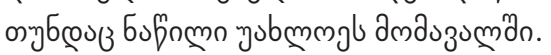

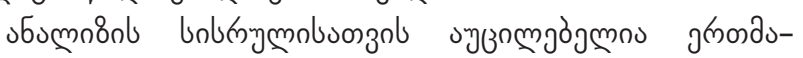

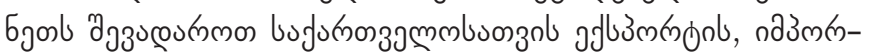

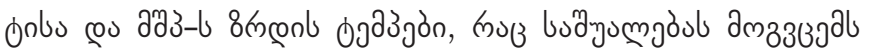

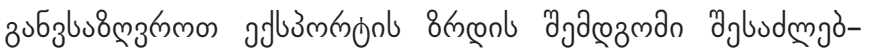
mmòjòn. nb. zbrnnmgòn 2 cos 3.

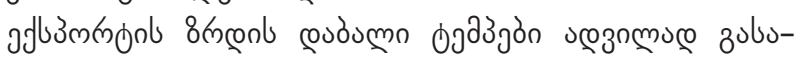

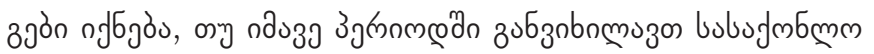

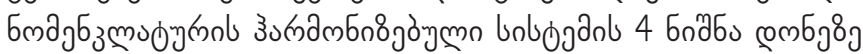

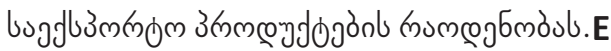

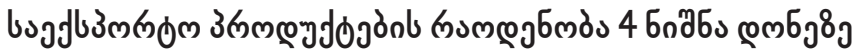

2012- 1641

$2013-1713$

$2014-1715$

$2015-1749$

2016-1735

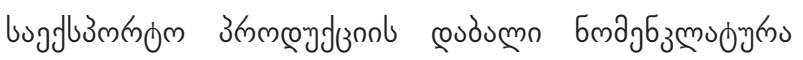

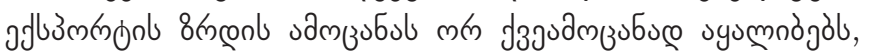

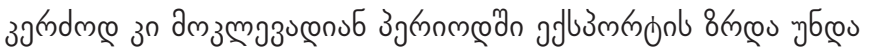

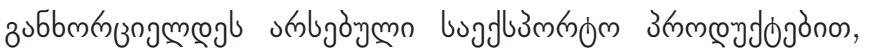

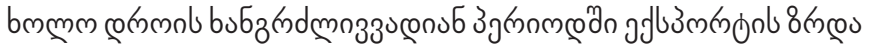

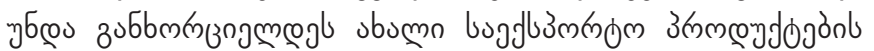

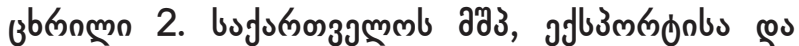

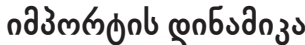

\begin{tabular}{|c|c|c|c|}
\hline & 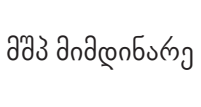 & 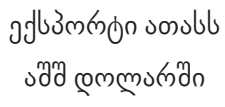 & 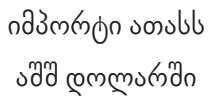 \\
\hline 2011 & 14.435 дмm & 2186 & 7072 \\
\hline 2012 & 15.846 ప mஙణ & 2377 & 8056 \\
\hline 2013 & 16.14 పмmळю & 2910 & 8023 \\
\hline 2014 & 16.509 дмாю & 2861 & 8602 \\
\hline 2015 & 13.994 పмm & 2205 & 7300 \\
\hline 2016 & 14.333 дмпю & 2113 & 7295 \\
\hline
\end{tabular}

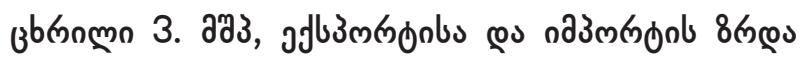

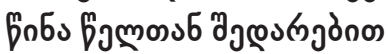

\begin{tabular}{|c|c|c|c|}
\hline & 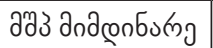 & aflusmmon & 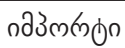 \\
\hline 2012 & 9,7 & 8,7 & 13,9 \\
\hline 2013 & 1,8 & 22,4 & $-0,5$ \\
\hline 2014 & 2,3 & $-1,7$ & 7,2 \\
\hline 2015 & $-15,3$ & $-23,0$ & $-15,2$ \\
\hline 2016 & 2,4 & $-4,2$ & $-0,01$ \\
\hline
\end{tabular}

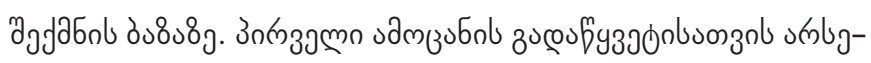

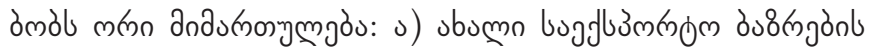

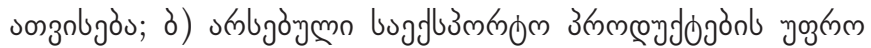

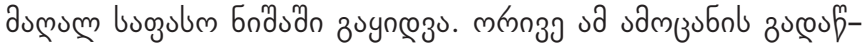

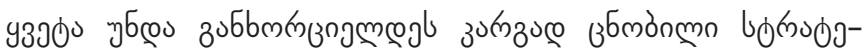

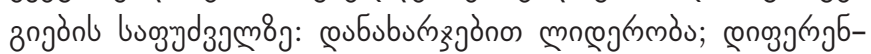

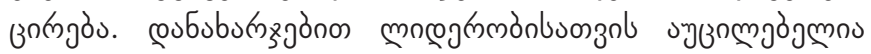

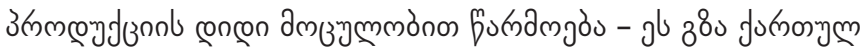

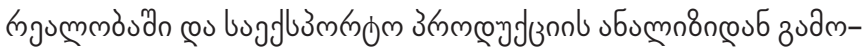

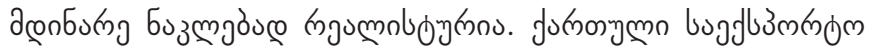

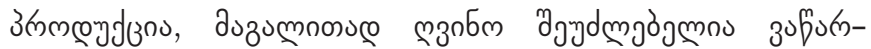

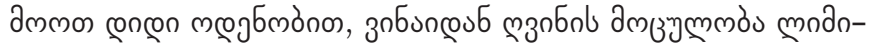

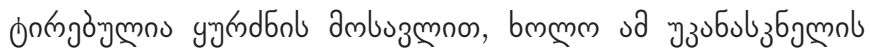

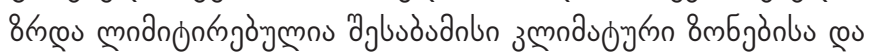

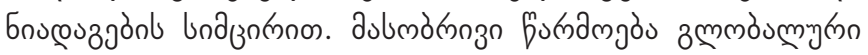

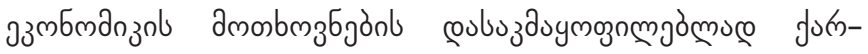

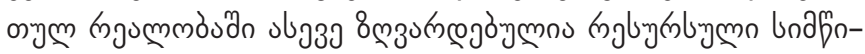

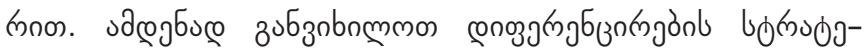

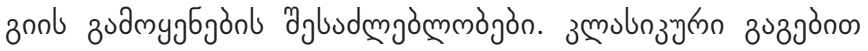
conoggr

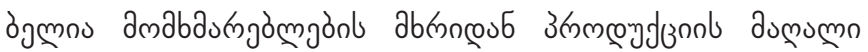

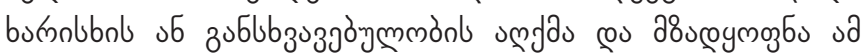

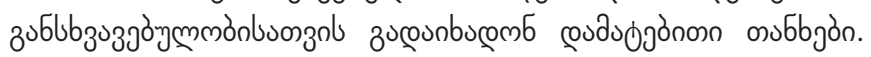

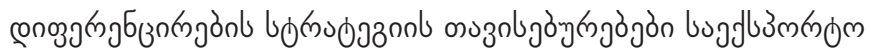

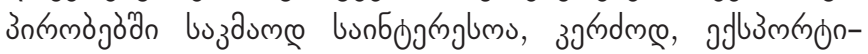




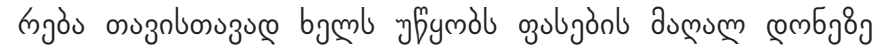

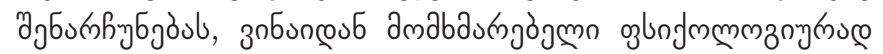

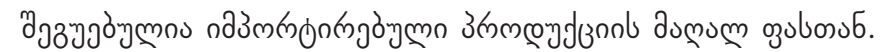

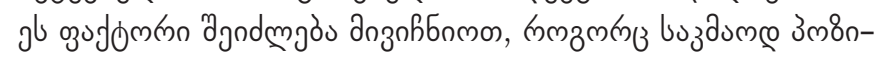
ongmo.

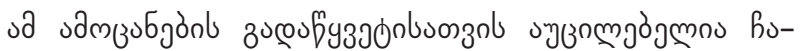

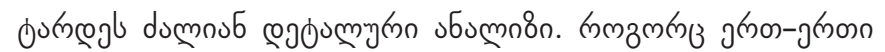

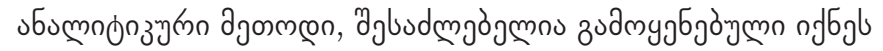

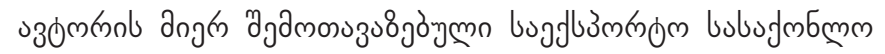

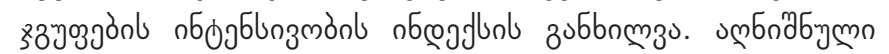

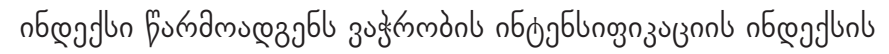
cos8inbogoub.

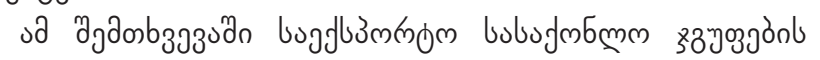

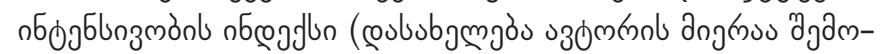

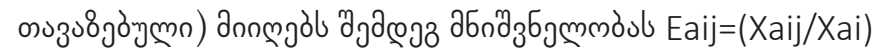
(Maj/Ma), bucoos:

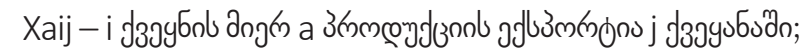

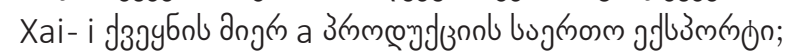

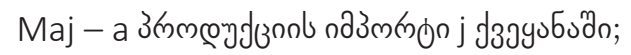

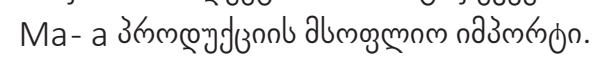

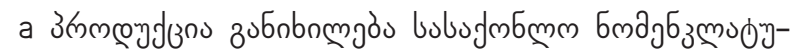

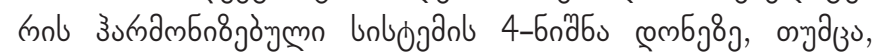

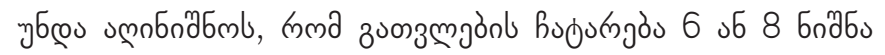

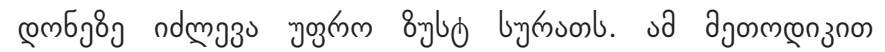

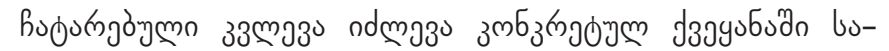

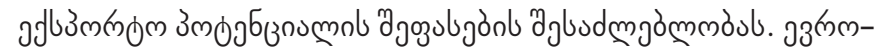

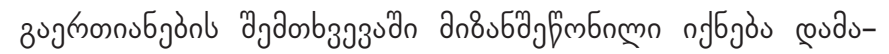

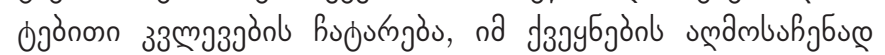

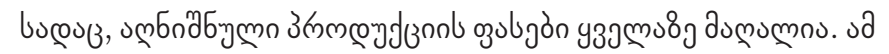

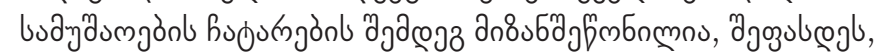

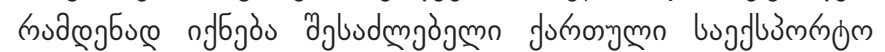

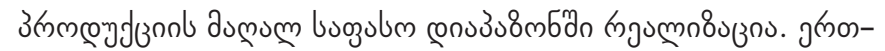

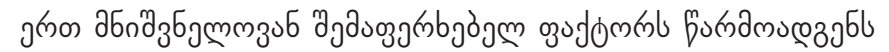

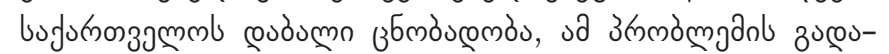
bufysaduc an8ubajpm mgònl zuamyjбgòu.

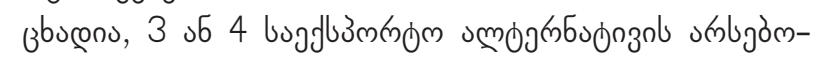

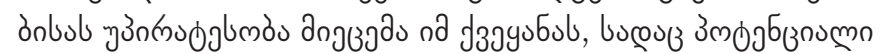

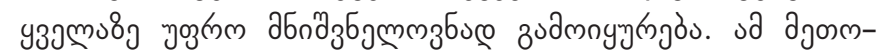

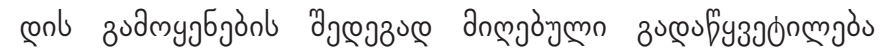

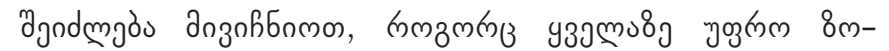

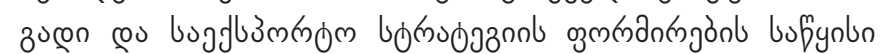

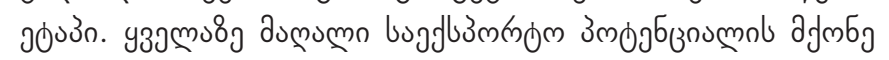

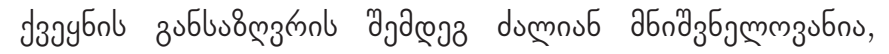

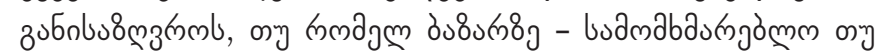

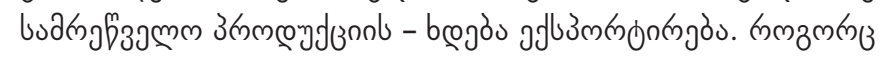

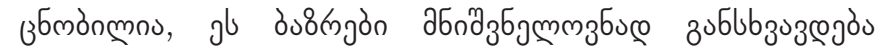

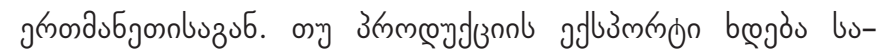

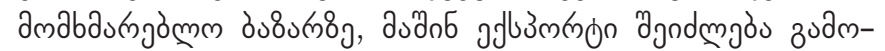

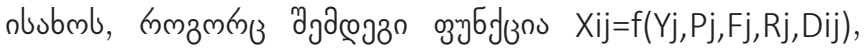

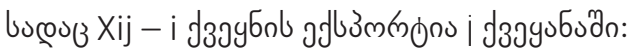

Yj - j J3aybol ambsobmgmós;

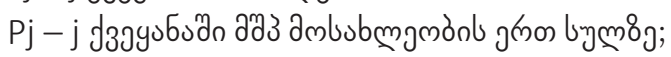

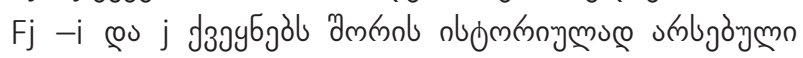

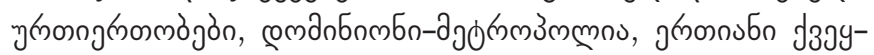

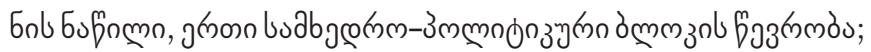

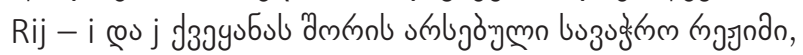

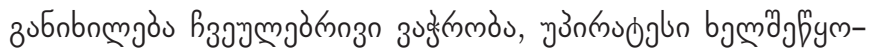

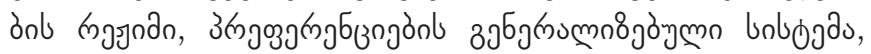

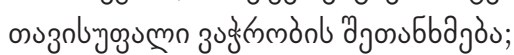

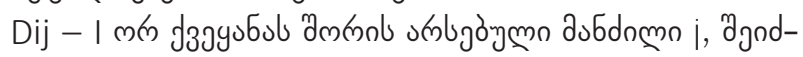

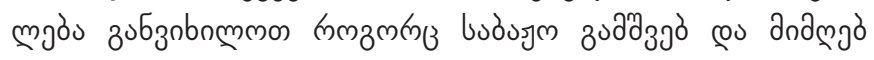

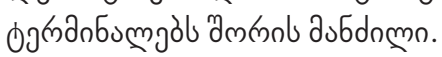

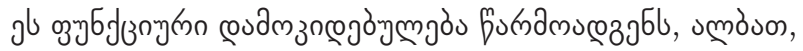
y3a mu

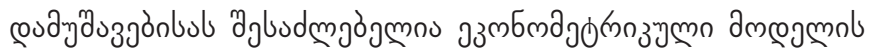
cosamaेozjos.

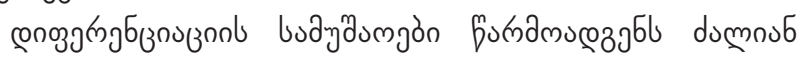

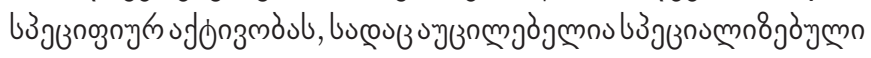

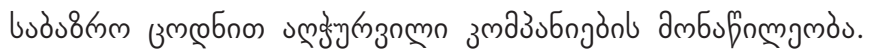

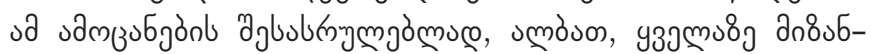

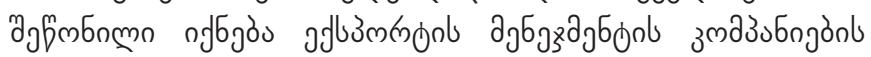

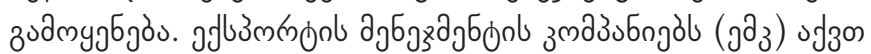

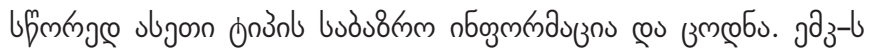

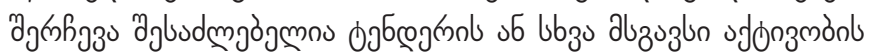

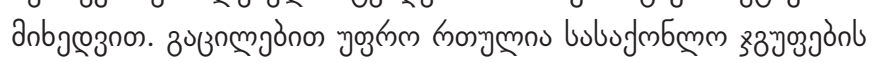

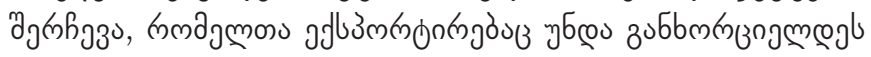

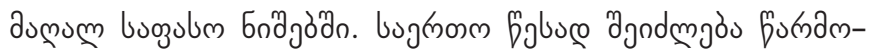
Зnœ

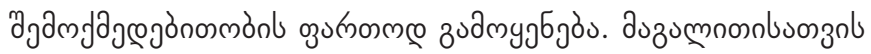

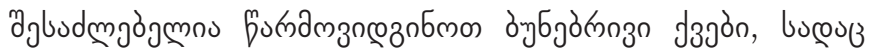

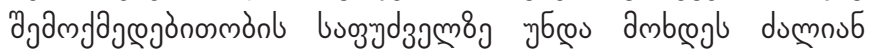

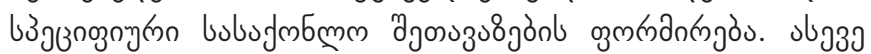

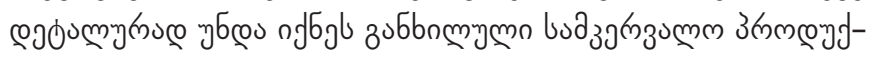

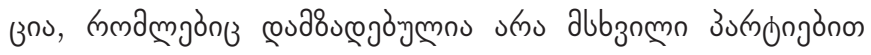

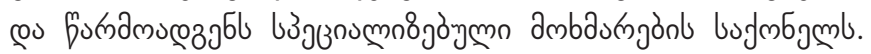

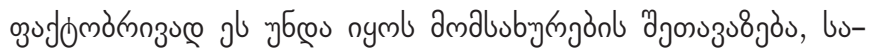

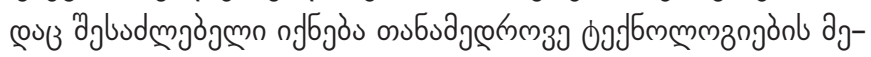

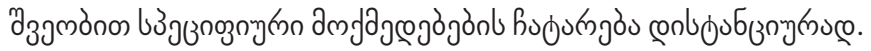

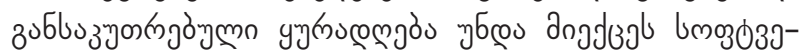

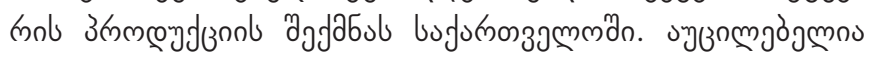

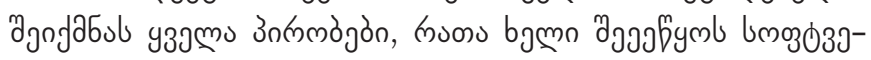

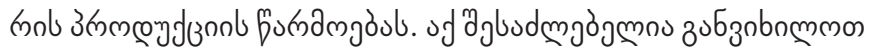

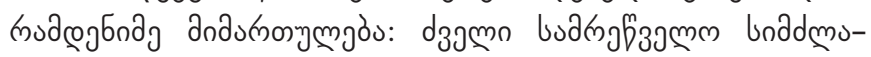

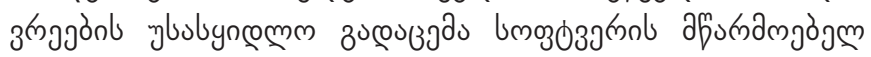

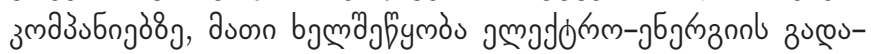

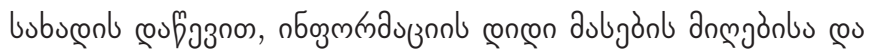

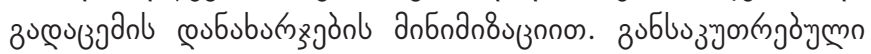




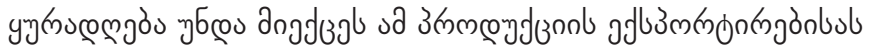

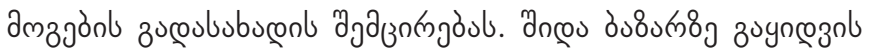

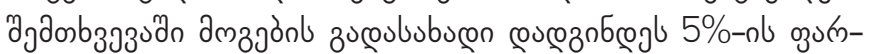

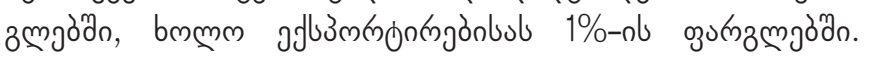

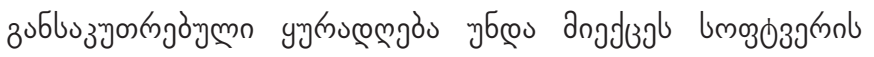

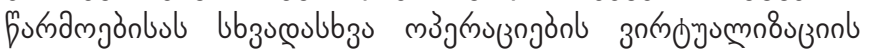

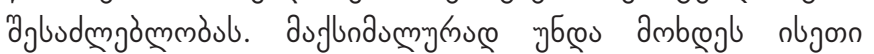

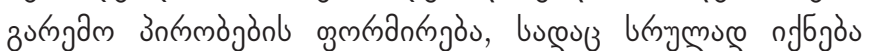

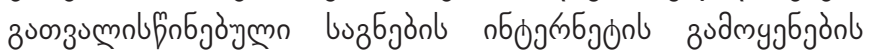

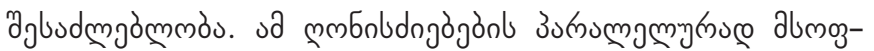

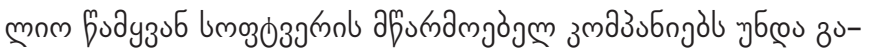

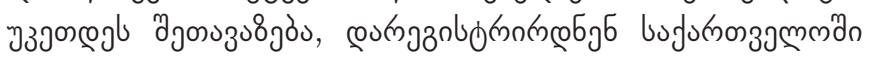

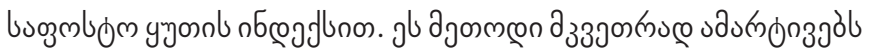

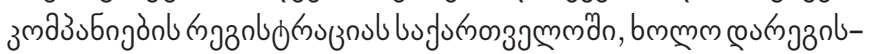

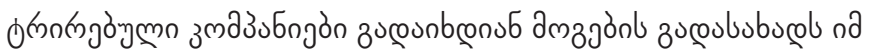

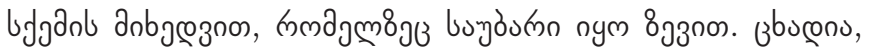

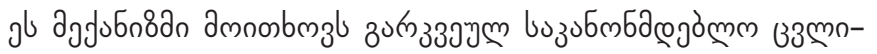

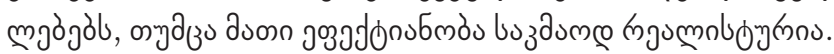

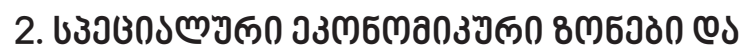

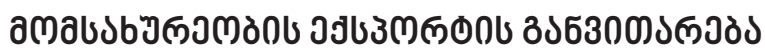

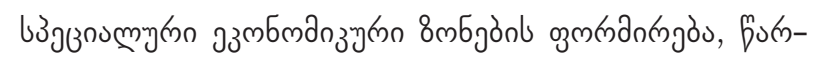

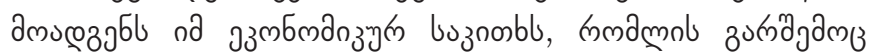

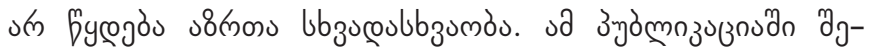

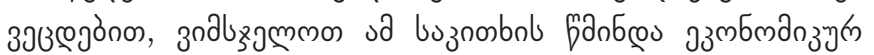

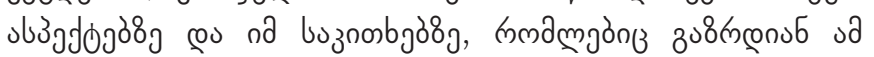

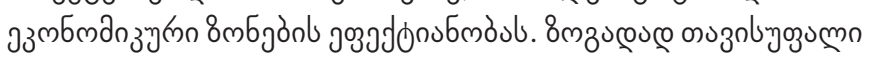

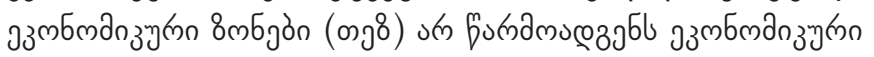

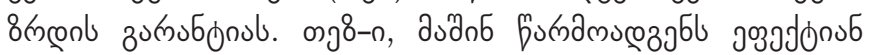

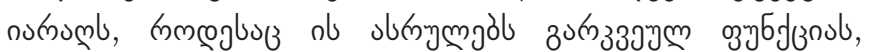

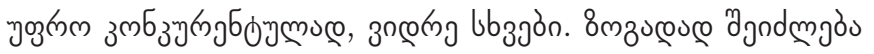

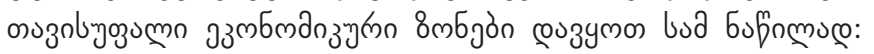

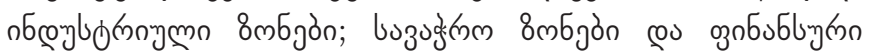

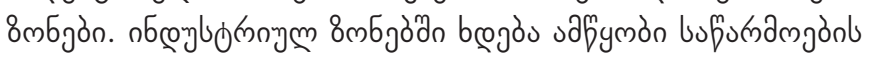

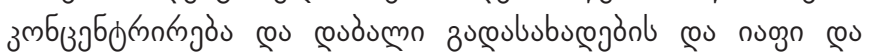

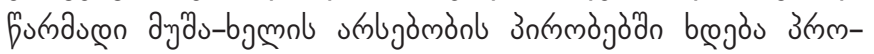

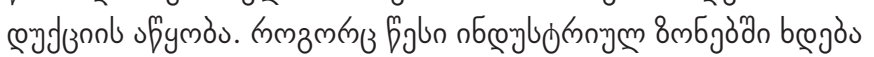

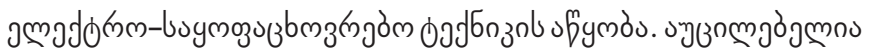

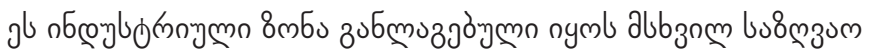

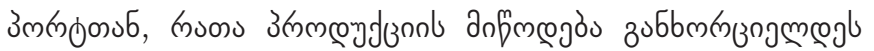

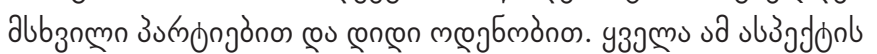

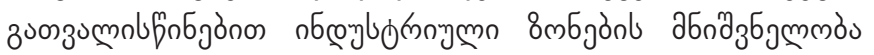

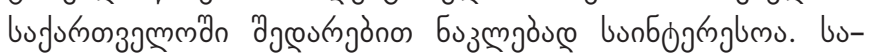

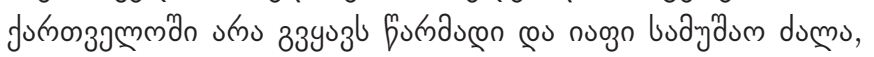

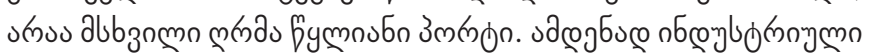

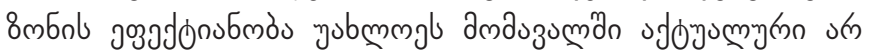
nftgos.

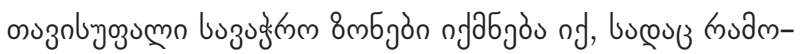

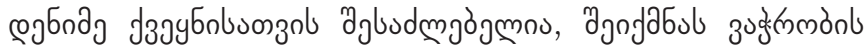

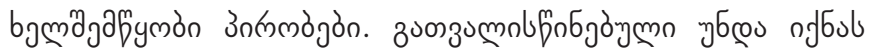

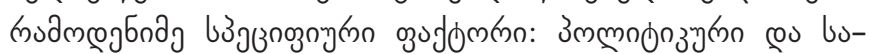

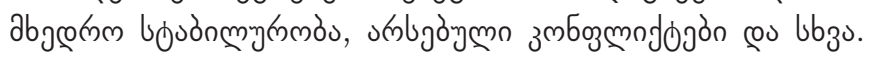

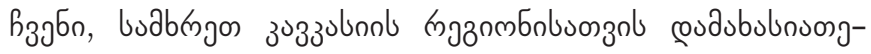

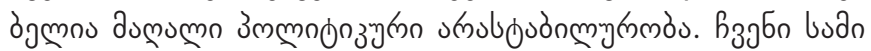

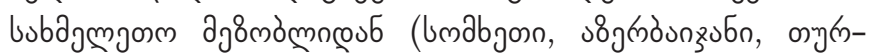
ذgon), abmmm

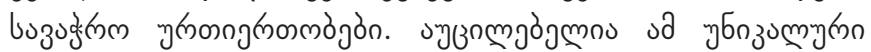

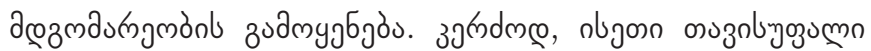

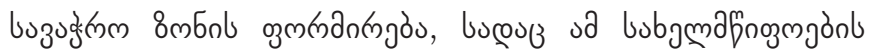

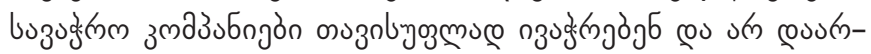

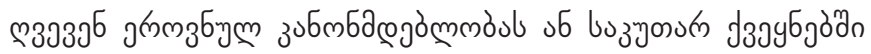

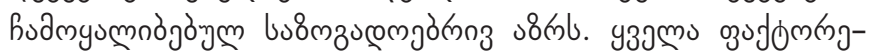

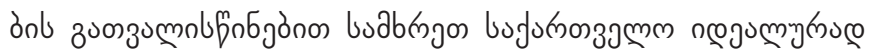

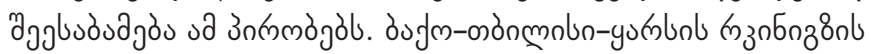

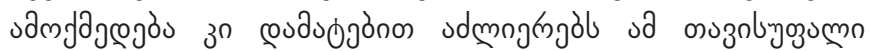

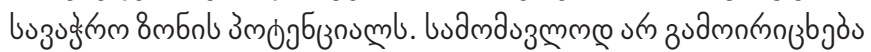

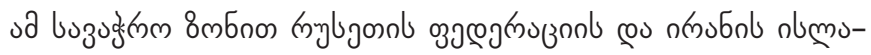

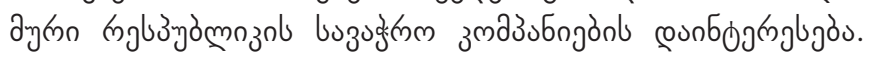
उ०सुक bud

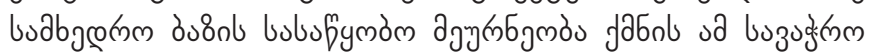

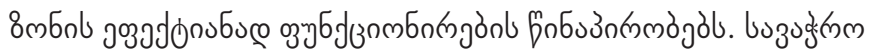

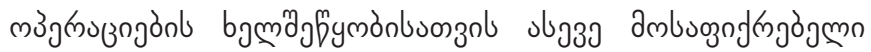

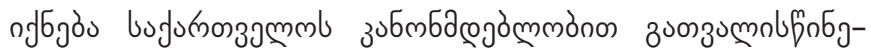

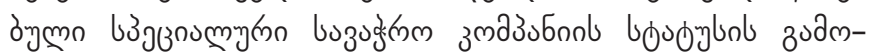

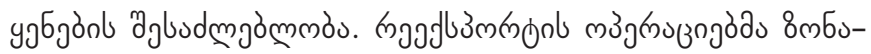

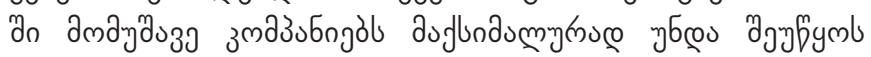

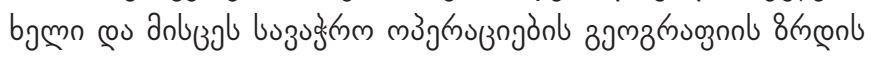

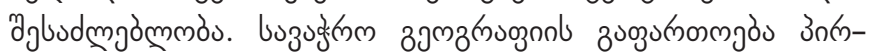

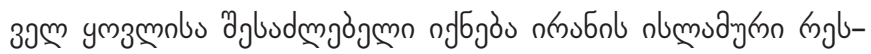

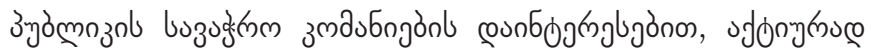

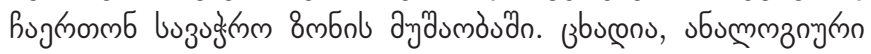

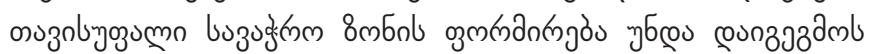

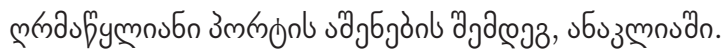

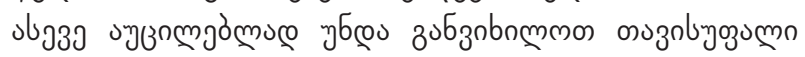

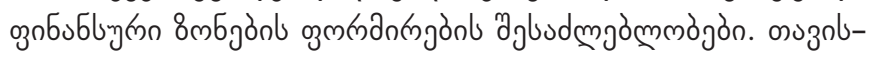

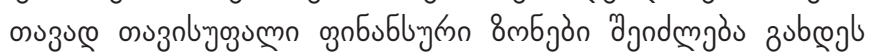

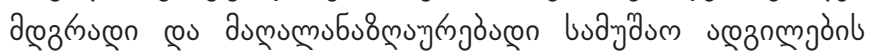

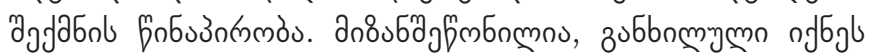

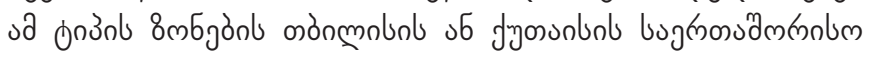

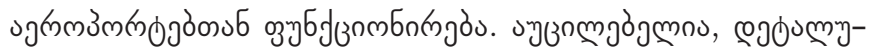

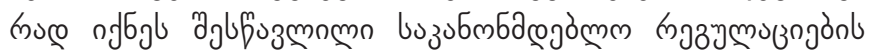

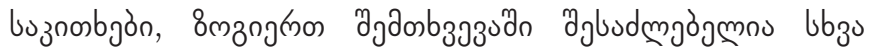

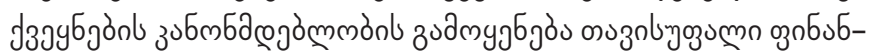

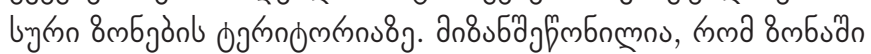

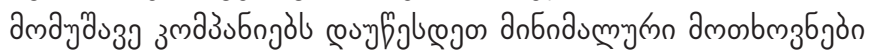

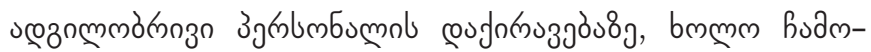

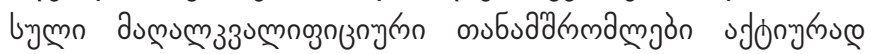




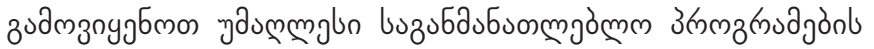

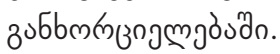

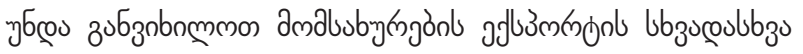

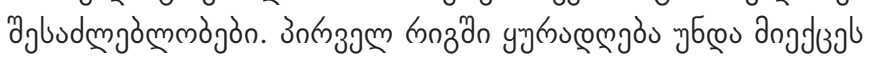

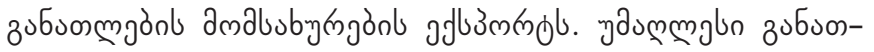

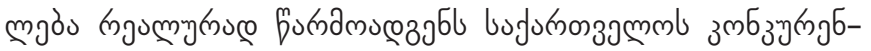

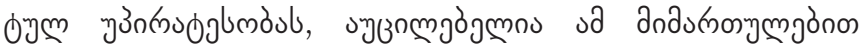

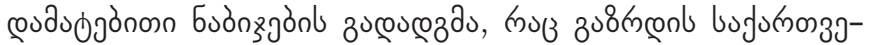

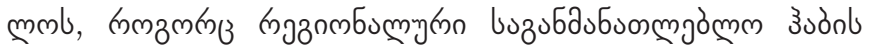

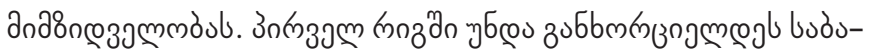

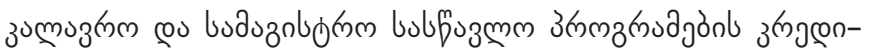

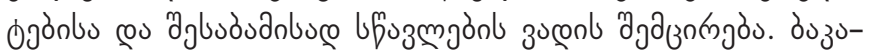

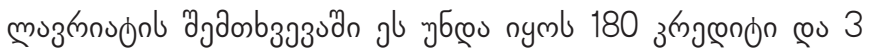

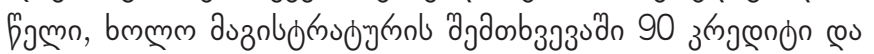

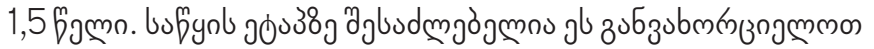

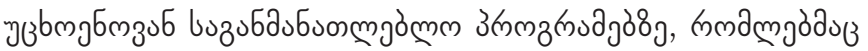

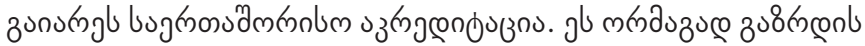

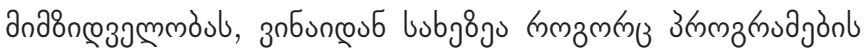

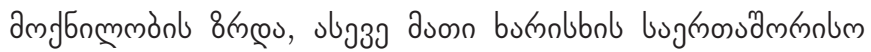
euscoubongrado.

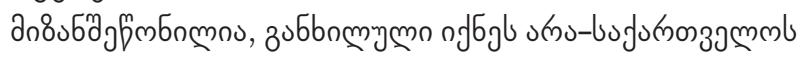

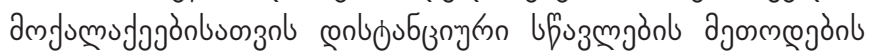

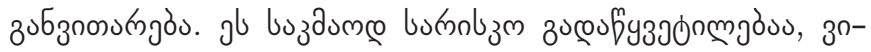

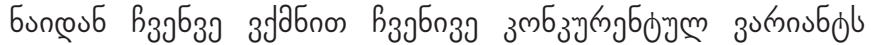

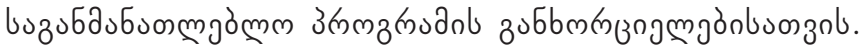

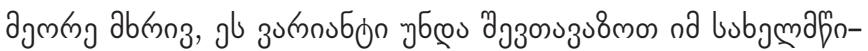

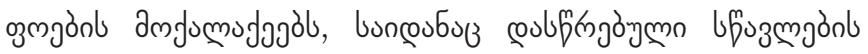

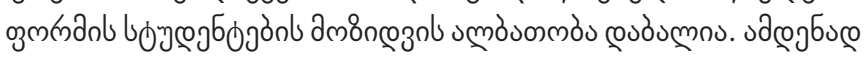

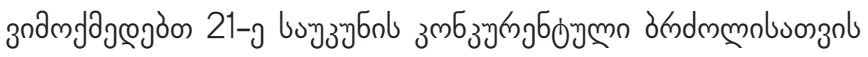

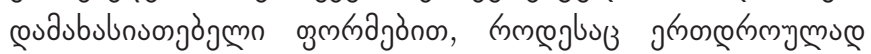

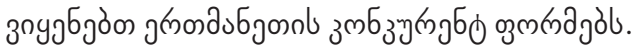

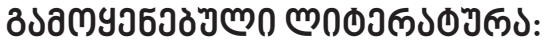

1. O'Cass, Aron and Craig Juliann (2003), “Examining Firm and Environmental Influences on Export Marketing Mix Strategy and Export Performance of Australian Exporters". European Journal of Marketing, 37 9314) 366-84

2. Papava, Vladimer, Retroeconomics - Moving from Dying to Brisk Economy, Journal of Reviews on Global economics, 2017, 6, pp 455-462

3. Papava, V. (2013). Economic Reforms in Post-Communist Georgia: Twenty Years After. New York: Nova Science Publishers.

4. Gaganidze, G.(2016). Georgian Export Potential Utilization on the EU Market, Journal of International Management Studies, Volume16, Number 1.

5. Gaganidze, G. (2015a). Export Potential and Competitive Advantage, scientific and practical journal, Economics and Business, N3.

6. Gaganidze, G. (2015b). Export Potential of Georgian Agricultural Products on the EU Market (Based on Competitive Advantages and Market Entry Modes), European Journal of Business Research, Volume 15, Number 2, ISSN326:1945-2977.

7. Gaganidze,G.(2014a) Competitive advantages of Georgian non-agricultural products on the EU Market, International Academic Conference, Business and Globalization, Dubai, 2-3rd February, ABRM, ISBN 2047-2854

8. Gaganidze, G. (2014b). Competitive Advantage of Georgian Agricultural Products on the EU Market, International Academic Conference on Economics, Management and Marketing in Prague, (IAC-EMM 2014) 7-8, August, Conference proceedings, ISBN 978-80-905442-6-0

9. Gaganidze,G. (2014c). Systematic Approach to Research the Competitive Advantage, TSU, scientific and practical journal, Economics and Business, N4, 2014

10. Gaganidze, G. (2014d). Determining the production export potential index, TSU, scientific and practical journal, Economics and Business, N1, January-February.

11. Gaganidze, G. (2013a). Competitiveness of Georgian agro products and creation of export strategies; II International Scientific-Practical Conference, Bioeconomy and Sustainable Development of Agriculture, October, Tbilisi, Proceedings

12. Gaganidze, G. (2013b). New Challenges in Managing International Companies, Global Business Conference Proceedings, ISSN 1848-2252;p.73.(on English)

13. Gaganidze, G. (2013c).Competitiveness of Georgian Agro products and creation of the export strategies on the EU market, TSU, scientific and practical journal, Economics and Business, N6November-December.

14. Silagadze, A., Zubiashvili, T. (2015). Parameters of the European Union and the Post-Soviet Georgia's Economy. Refereed International Journal of Business and Management Studies (IJBMS), pp. 441-448.

15. Silagadze, A., Atanelishvili,T. (2014). The main economic indicators of the EU and Georgia "Topical problems of the development of economy and economic science." Collection of scholarly works of Paata Gugushvili Institute of Economics TSU, pp.50-5 


\section{EXPORT ISSUES IN ASSESSING COUNTRY'S COMPETITIVENESS}

\section{GIORGI GAGANIDZE}

https://doi.org/10.35945/gb.2018.06.003

Doctor of Economics, Professor

Ivane Javakhishvili Tbilisi State University, Georgia

Giorgi.gaganidze@tsu.ge

KEY WORDS: COMPETITIVE ADVANTAGE, EXPORT PRODUCTION

\section{SUMMARY}

Often in scientific circles, mistrust regarding the reliability of competitiveness or other indices is expressed. The definite part of the economists think that it is not advisable to form any serious strategies based on these indices. This mistrust is probably caused by less awareness in these issues, especially because of the absence of tangible, confirmed ties between these indices and the real economic figures. We often witness only superficial talks about indices, focusing on the place of the particular index of the country in certain rankings, which causes its politicization and the discussion of the fundamental issues is not taken into account. In this publication we will try to concentrate on the indices focusing on country's competitiveness and trade support. Such a choice was determined by two important issues: the first is the direct connection of these indices with the competitiveness of the country's exports and the second - both of these indices are made by the World Economic Forum and hence certain unified methodological approaches exist. 